\title{
Signature of a Chemical Bond in the Conductance between Two Metal Surfaces
}

\author{
W. A. Hofer* \\ Surface Science Research Centre, University of Liverpool, Liverpool L69 3BX, United Kingdom
}

A. J. Fisher

Department of Physics and Astronomy, University College, Gower Street, London WC1E 6BT, United Kingdom

(Received 21 October 2002; published 17 July 2003)

\begin{abstract}
Conductance in monatomic metal contacts is quantized; it increases in discrete steps of one conductance quantum $2 e^{2} / h$. By contrast, in a vacuum barrier between two metal surfaces we find that conductance increases linearly and continuously with the interaction energy between individual atoms. This behavior shows unambiguously that current flow between single atoms is a measure for their chemical interaction. In the controlled environment of a scanning tunneling microscope it should allow us to study the formation of covalent bonds up to the point where these atoms finally jump into contact.
\end{abstract}

DOI: 10.1103/PhysRevLett.91.036803

PACS numbers: 73.40.Gk, 71.15.Mb, 73.23.Hk

Because of their importance for technical applications the conductance properties of metals have been widely researched. In metal break junctions at close to zero temperature the conductance of single atomic contacts has been measured with great accuracy, and it was generally found that conductance increases in discrete steps of one conductance quantum $2 e^{2} / h$ [1-5]. The rupture of a bond probes into material properties from the starting point of material stability. Quite the opposite is true for the operation of scanning probe microscopes. There, the interaction between two separate surfaces is generally kept at such a low level that the small changes in the forces between the surfaces or in the number of electrons tunneling through the vacuum barrier can be used as an indication of the surface topographic and electronic properties. The intermediate range between these two methods continues to be problematic. In particular, the role of chemical interactions in scanning probes, even though widely researched [6-13], is still not well understood. Part of the problem is the theoretical representation of the situation in different frameworks: perturbation methods are generally limited to high distances, they can incorporate chemical interactions only from the outset [13-15]. Scattering methods, while electronically accurate, cannot treat atomic interactions and their shift of position at low distances [16]. Nonequilibrium methods are in principle reliable, but they cannot simulate actual probe scans due to their innate restrictions of the symmetry of the combined system [17,18].

Here, we propose a new method, which incorporates chemical interactions in a natural way. The method is based on extensive ab initio calculations of coupled systems. These calculations, including chemical interactions from the outset, establish that the suggested perturbation method is in general sufficient to reach the highest level of experimental accuracy.

A system, composed of a conducting surface and an equally conducting probe, will be completely decoupled if the distance between the surface atoms and the foremost atom of the probe (apex atom) is substantially greater than $1 \mathrm{~nm}$. Then the electron states of surface and probe are orthogonal: every product

$$
\left(\chi_{\nu}^{*}, V \psi_{\mu}\right):=\int d^{3} r \chi_{\nu}^{*}(\mathbf{r}) V(\mathbf{r}) \psi_{\mu}(\mathbf{r})
$$

will be zero. If the two systems are brought into closer contact, with a distance of about $0.5-0.6 \mathrm{~nm}$, the presence of the other lead will have an effect on the electronic structure and electron dynamics on both sides. The two systems in this case are weakly coupled, the change of the physical situation compared to the high distance range can be described by a perturbation potential $V$. The two main effects occurring in this range are as follows.

(i) A transition of electrons from one side to the other, the transition rate given by Fermi's golden rule [19],

$$
\Gamma_{\mu \nu}=\frac{2 \pi}{\hbar}\left|\left(\chi_{\nu}^{*}, V \psi_{\mu}\right)\right|^{2} \delta\left(E_{\nu}-E_{\mu}\right) .
$$

This relation is equivalent to Bardeen's formulation of the tunneling problem $[14,15]$. The reason for using the Bardeen formulation rather than Fermi's golden rule in the calculation of tunneling currents [20] is a technical one: the perturbation potential due to the approach of a surface and a probe is commonly unknown.

For a finite system with a discrete set of eigenvalues, or for nonzero temperatures, the delta function has to be replaced by a smeared-out function, for example, a Gaussian of half-width $\sigma$; thus the tunneling current $I_{\mu \nu}=e \Gamma$ for a single transition is described by

$$
I_{\mu \nu}=\frac{2 \pi e}{\hbar \sigma \sqrt{\pi}}\left|\left(\chi_{\nu}^{*}, V \psi_{\mu}\right)\right|^{2} e^{-\left(E_{\nu}-E_{\mu}\right)^{2} / \sigma^{2}} .
$$

(ii) The second effect is a change of the system energy. In a second order perturbation expansion, the change in the energy of a filled state $\chi_{\nu}$ is given by 


$$
\begin{aligned}
-\Delta E_{\nu}^{(2)}= & \left(\chi_{\nu}^{*}, V \chi_{\nu}\right)+\sum_{\lambda} \frac{\left|\left(\chi_{\nu}^{*}, V \chi_{\lambda}\right)\right|^{2}}{E_{\nu}-E_{\lambda}} \\
& +\sum_{\lambda} \frac{\left|\left(\chi_{\nu}^{*}, V \psi_{\lambda}\right)\right|^{2}}{E_{\nu}-E_{\lambda}}
\end{aligned}
$$

where the sum goes in principle over all empty states $\chi_{\lambda}$ and $\psi_{\lambda}$. The first term is the change of the eigenvalue due to the coupling potential $V$, the second term describes the changes due to transitions between states on one side of the barrier only, while the final term describes transitions across the tunneling barrier between tip and sample states. Only the third type will contribute to the interaction energy between the two surfaces, because the wave functions and the potential are exponentially decreasing and the perturbing potential for states of the tip is the potential of the sample surface. Focussing on the energy contribution due to a single pair of states $(\mu, \nu)$, we obtain within perturbation theory

$$
-\Delta E_{\mu \nu}=\frac{\left|\left(\chi_{\nu}^{*}, V \psi_{\mu}\right)\right|^{2}}{E_{\nu}-E_{\mu}} .
$$

Comparing Eqs. (5) and (3) we find for the relation between the current $\Delta I_{\mu \nu}$ and interaction energies $\Delta E_{\mu \nu}$ the following expression:

$$
\Delta I_{\mu \nu}=\frac{2 \pi e}{\hbar \sigma \sqrt{\pi}}\left(E_{\nu}-E_{\mu}\right) e^{-\left(E_{\nu}-E_{\mu}\right)^{2} / \sigma^{2}} \Delta E_{\mu \nu} .
$$

Note that interaction energies contribute a negative term to the total energy of interacting systems. But to compare with the (positive) tunneling current we use their absolute values in the rest of the Letter. In this formulation the tunneling current from a single transition $\left(\Delta I_{\mu \nu}\right)$ appears to be proportional to its contribution to the interaction energy $\left(\Delta E_{\mu \nu}\right)$. Because of level broadening, the energy difference $E_{\nu}-E_{\mu}$ is of the same scale as $\sigma$. Consequently, we set $E_{\nu}-E_{\mu} \approx \sigma$. Then the above relation gives

$$
\Delta I_{\mu \nu} \approx \frac{2 e \sqrt{\pi}}{2.718 \hbar} \Delta E_{\mu \nu} \approx 4 \frac{2 e}{h} \Delta E_{\mu \nu} .
$$

Interestingly, the relation then is very similar to the Landauer-Büttiker formulation of the tunneling problem [16]. Although the right-hand side of Eq. (6) does not appear to contain the transition matrix $T$, it implicitly appears because $T$, like the interaction energy $\Delta E_{\mu \nu}$, is proportional to $\left|\left(\chi_{\nu}^{*}, V \psi_{\mu}\right)\right|^{2}$. Consequently, as shown by Feuchtwang and others [17], the perturbation treatment is the lowest-order term in a full scattering treatment of the transport. Within this approximation the equation amounts numerically to

$$
\Delta I_{\mu \nu}[\mathrm{nA}] \approx 3.1 \times 10^{5} \Delta E_{\mu \nu}[\mathrm{eV}] .
$$

However, our numerical results, and experimental evidence (see below) suggest that the proportionality be- tween net current and total interaction energy holds even beyond the domain of validity of this approximation.

This may be understood by writing the interaction energy as

$$
E_{\mathrm{int}}=\operatorname{Tr}[\rho V]=-\frac{1}{\pi} \int_{-\infty}^{\mu} G^{r}(E) V d E,
$$

where $\rho$ is the one-electron density matrix and $G^{r}$ the retarded one-electron Green's function. The part of $E_{\text {int }}$ coming from the mixing of tip and sample states involves the off-diagonal elements of $\rho$ (and hence of $G^{r}$ ) linking tip and sample. These are determined by Dyson's equation, and hence to lowest order in $V$,

$$
G^{r}=G_{0}^{r}+G_{0}^{r} V G_{0}^{r} .
$$

Thus the interaction energy to lowest order is

$$
E_{\mathrm{int}}=-\frac{1}{\pi} \int_{-\infty}^{\mu} \operatorname{Tr}\left[G_{0}^{r} V G_{0}^{r} V\right],
$$

which (like the total current) is quadratic in $V$.

The theoretical result suggests that the current is linear with the interaction energy between the two surfaces. The observation is supported by experimental evidence. Interaction energies are not directly accessible in experiments, but the attractive force between the two surfaces and the tunneling current can be simultaneously determined. In combined experiments it is found that current and forces follow the same characteristic curve during an approach [6,12]. In the recent experiments by Schirmeisen et al. [12] the square of the force during an approach shows a parabolic behavior in the low conductance regime, while it is close to linear in the high regime (their Fig. 5). If long range forces were determined by chemical interactions, this feature would be observable even for distances above $6 \AA$. In this range, however, forces are mainly due to dispersion interactions, which do not decay exponentially [21].

In our simulations we have employed a pseudopotential density functional method [22,23], using a $3 \times 3$ surface unit cell and a pyramid for the STM tip. Not including relaxation effects, which have a very limited range, the tunneling current is described by a perturbation approach using the atomic arrangement of the noninteracting subsystems [20]. Interaction energies are determined by relaxing the atomic positions of the coupled system of surface and tip to their energetic minimum at selected points during an approach. We have simulated two separate experiments: the approach of a tungsten tip onto a $\mathrm{Au}(111)$ surface, and the approach of $\mathrm{Cu}$-contaminated tip onto a $\mathrm{Cu}(100)$ surface. The technical details of the calculation have been described in our previous work [13].

We have calculated the current for a very low bias voltage of $-1 \mathrm{mV}$, interaction energy and tunneling conductance are shown in Fig. 1. From the onset of chemical forces, at a distance of about $0.55 \mathrm{~nm}$, to the point where 


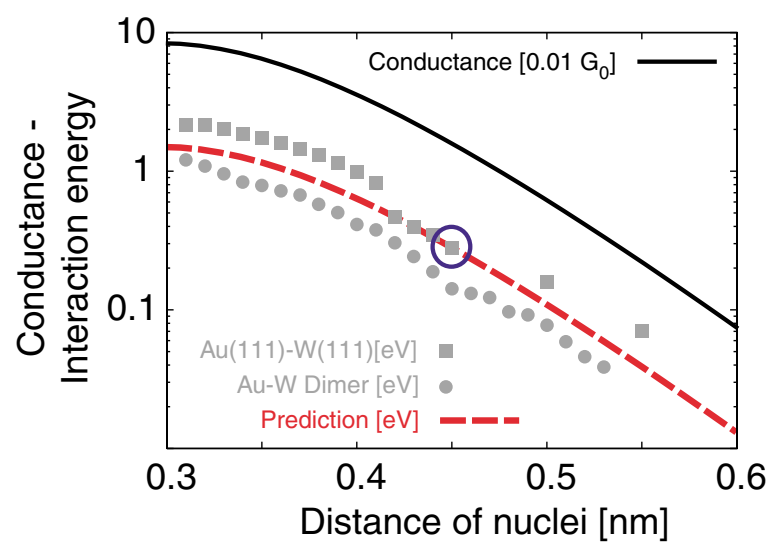

FIG. 1 (color online). Interaction energy (solid squares), tunneling conductance (black curve) in units of $G_{0}=2 e^{2} / h$, the conductance quantum, and predicted interaction energy (dashed curve) in an $\mathrm{Au}(111)-\mathrm{W}(111)$ system. The predicted interaction energy over the whole distance range has been determined from a single point of the calculation for the coupled system (empty circle). Interaction energies between $\mathrm{Au}$ and $\mathrm{W}$ atoms in a dimer (solid circles) are given for comparison.

the tip breaks at $0.41 \mathrm{~nm}$, the interaction energy is nearly proportional to the conductance. We attribute the deviations from the linear characteristic to relaxations of surface and tip atoms, which distort the result (see below). The linearity between conductance and interaction energy has been verified by an additional calculation of a $\mathrm{Cu}(100)$ system. This simulation establishes that the feature is independent of the chemical composition or the orientation of a surface. The STM tip was mimicked by a square pyramid of two layers on a five-layer $\mathrm{Cu}(100)$ film. The result of the simulation is shown in Fig. 2. Also in this case the relation between interaction energy and conductance is generally linear. We note slight deviations of the linearity in two distance ranges. In the range above $0.5 \mathrm{~nm}$ the interaction energies between $\mathrm{Cu}$ atoms of sample and tip are very small, the absolute error in a total energy calculation becomes therefore disproportionately large. In the range below $0.35 \mathrm{~nm}$ the shift of atomic positions due to interactions remains unconsidered in the current calculation.

The predicted interaction energy for the $\mathrm{Au}(111)$ system is shown in Fig. 1. The constant of proportionality has been estimated from the point where surface and tip were $0.45 \mathrm{~nm}$ apart. With the exception of the range where atoms are substantially displaced, indicating the loss of elastic properties of surface atoms, the prediction agrees well with the result from first principles calculations of the coupled system. An identical calculation has been performed for the $\mathrm{Cu}(100)$ system. The predicted interaction energy is shown in Fig. 2. Also in this case the agreement between the perturbation method and the first principles calculation of the coupled system seems acceptable. The proportionality factor between conductance

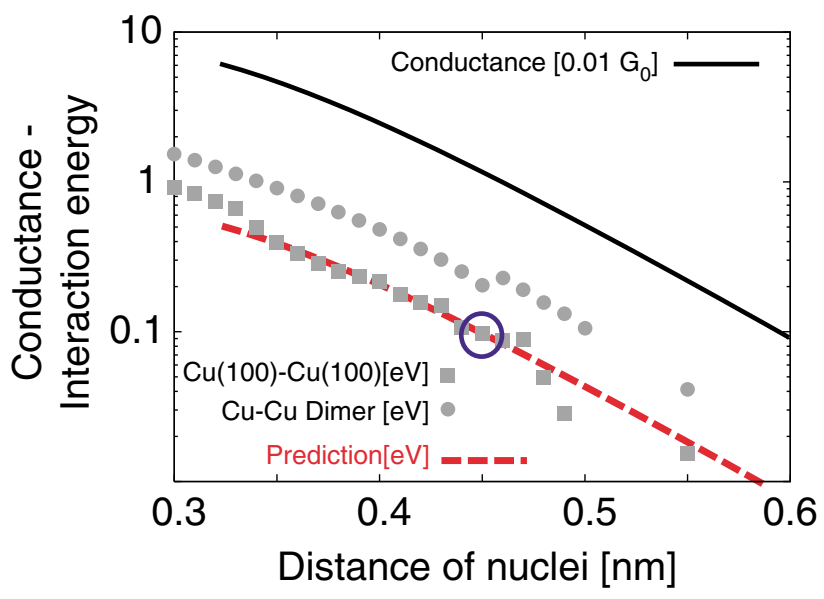

FIG. 2 (color online). Interaction energy, tunneling conductance, and predicted interaction energy in a $\mathrm{Cu}(100)-\mathrm{Cu}(100)$ system. The interaction energies between $\mathrm{Cu}$ atoms in a dimer are given for comparison.

and interaction energy depends on the system under consideration. For the $\mathrm{Au}(111)$ and the $\mathrm{W}$ tip (5d elements) we obtain $0.25\left[\mathrm{eV} / \mathrm{M} \Omega^{-1}\right]$, the corresponding factor for the $\mathrm{Cu}(100)$ surface ( $3 d$ elements) is $0.12\left[\mathrm{eV} / \mathrm{M} \Omega^{-1}\right]$. For higher bias voltages the conductance will be reduced because the condition of elastic tunneling at room temperature entails in every case a thermal broadening of the energy range to about $0.1 \mathrm{eV}$. Thus the current does not increase substantially for changes of the bias voltage from -1 to $-50 \mathrm{mV}$, whereas conductance decreases quite dramatically. The loss of elastic properties of surface and tip systems due to a critical limit of conductance in very low bias scans occurs at values of $2.5[\mathrm{Au}(111)]$ and $5.0[\mathrm{Cu}(100)] \mathrm{M} \Omega^{-1}$. The result is in good agreement with the experimental observation of "roll-on" effects of atomic corrugations observed on $\mathrm{Cu}(100)$ [9]. The difference observed for two different tips seems to correspond to the different decay of $5 d$ and $3 d$ electrons into the vacuum. The current in the electronic circuit of the instrument under these conditions is typically less than $10 \mathrm{nA}$. The distance between tip and surface in this case is about $4 \AA$, with an interaction energy of about $1 \mathrm{eV}$. The numerical estimate Eq. (8) shows that the actual current in the vacuum barrier, which is responsible for chemical interactions, must be much higher and close to $10^{5} \mathrm{nA}$. The difference derives from the fact that the main part of this current is not unidirectional, but bidirectional.

Finally, we have calculated the attractive forces from the derivative of interaction energies. In this calculation the predictions for the interaction energy based on a single point of the energy curve during an approach were numerically differentiated. These results are shown in Figs. 3(a) and 3(b). Comparing with the forces of the coupled systems, good agreement is obtained up to the jump into contact [at about $0.41 \mathrm{~nm}$ for $\mathrm{Au}(111)$ [13] and about $0.34 \mathrm{~nm}$ for $\mathrm{Cu}(100)$ ]. The figures show that 


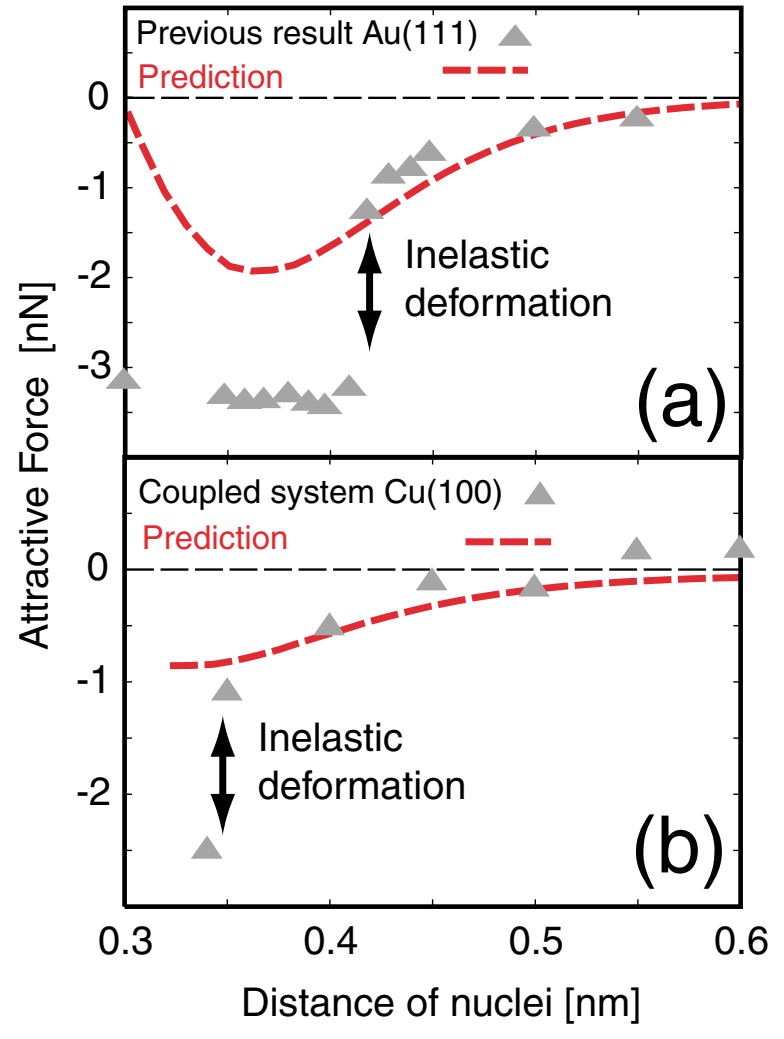

FIG. 3 (color online). Attractive forces between the surfaces. (a) Predictions for the $\mathrm{Au}(111)$ surface (dashed line) are compared with previous calculations (solid triangles) [13]. Up to the jump into contact, indicated by the sudden onset of large displacements (see arrows), the predicted force is equal to the force calculated from the coupled system. (b) An identical behavior is observed for the $\mathrm{Cu}(100)$ surface. The jump into contact (see arrows) occurs at a lower distance due to the different decay of $5 d$ and $3 d$ states.

perturbation theory can even be used to estimate the limit of stability: in both cases the jump into contact occurs when the forces reach a level of $1-1.5 \mathrm{nN}$. The method should prove valuable in determining the chemical forces in atomic force microscopy (AFM). Thus far, a perturbation method to treat these forces, which are at the bottom of atomic resolution [24,25], has proved elusive. As shown here, these forces can be calculated from the overlap of the wave functions of the decoupled systems and a single point of the energy curve of the coupled system. The remaining tasks, for the simulation of AFM images, are the determination of long range forces and elastic deformations.

The authors acknowledge helpful discussions with R. Wolkow and P. Grütter. This work was supported by the British Council, the National Research Council of Canada, and the IRC in Nanotechnology.
*Electronic address: whofer@liverpool.ac.uk

[1] J. M. Krans, C. J. Muller, I. K. Yanson, Th. C. M. Govaert, R. Hesper, and J. M. van Ruitenbeek, Phys. Rev. B 48, 14721 (1993).

[2] G. Rubio, N. Agrait, and S. Vieira, Phys. Rev. Lett. 76, 2302 (1996).

[3] A. I. Yanson, G. R. Bollinger, H. E. van den Brom, N. Agrait, and J. M. van Ruitenbeek, Nature (London) 395, 783 (1998).

[4] H. Ohnishi, Y. Kondo, and K. Takayanagi, Nature (London) 395, 780 (1998).

[5] E. Scheer, N. Agrait, J. C. Cuevas, A. L. Yegati, B. Ludoph, A. Martin-Rodero, G. R. Bollinger, J. M. van Ruitenbeek, and C. Urbina, Nature (London) 394, 154 (1998).

[6] U. Dürig, J. K. Gimzewski, and D.W. Pohl, Phys. Rev. Lett. 57, 2403 (1986).

[7] J. K. Gimzewski and R. Moller, Phys. Rev. B 36, 1284 (1987).

[8] U. Dürig, O. Züger, and D.W. Pohl, Phys. Rev. Lett. 65, 349 (1990).

[9] A. R. H. Clarke, J. B. Pethica, J. A. Nieminen, F. Besenbacher, E. Laegsgaard, and I. Stensgaard, Phys. Rev. Lett. 76, 1276 (1996).

[10] L. Olesen, M. Brandbyge, M. R. Sorensen, K.W. Jacobsen, E. Laegsgaard, I. Stensgaard, and F. Besenbacher, Phys. Rev. Lett. 76, 1485 (1996).

[11] G. Cross, A. Schirmeisen, A. Stalder, P. Grütter, M. Tschudy, and U. Dürig, Phys. Rev. Lett. 80, 4685 (1998).

[12] A. Schirmeisen, G. Cross, A. Stalder, P. Grütter, and U. Dürig, New J. Phys. 2, 29 (2000).

[13] W. A. Hofer, A. J. Fisher, R. A. Wolkow, and P. Grütter, Phys. Rev. Lett. 87, 236104 (2001).

[14] J. Bardeen, Phys. Rev. Lett. 6, 57 (1961).

[15] J.C. Chen, Introduction to Scanning Tunneling Microscopy (Oxford University Press, Oxford, 1993).

[16] M. Büttiker, Y. Imry, R. Landauer, and S. Pinhas, Phys. Rev. B 31, 6207 (1985).

[17] T. E. Feuchtwang, Phys. Rev. B 13, 517 (1976), and references therein.

[18] M Brandbyge, J. L. Mozos, P. Ordejon, J. Taylor, and K. Stokbro, Phys. Rev. B 65, 165401 (2002).

[19] C. Kittel, The Quantum Theory of Solids (Wiley, New York, 1963).

[20] W. A. Hofer and J. Redinger, Surf. Sci. 447, 51 (2000).

[21] H. Rydberg, B. I. Lundqvist, D. C. Langreth, and M. Dion, Phys. Rev. B 62, 6997 (2000).

[22] G. Kresse and J. Hafner, Phys. Rev. B 48, 13115 (1993).

[23] G. Kresse and J. Furthmüller, Phys. Rev. B 54, 11169 (1996).

[24] C. Barth and M. Reichling, Nature (London) 414, 54 (2001).

[25] A. S. Foster, C. Barth, A. L. Shluger, and M. Reichling, Phys. Rev. Lett. 86, 2373 (2001). 